Richard Allan Gadelha de Freitas ${ }^{1}$

Isabel Karolyne Fernandes Costa ${ }^{1}$

Rodrigo Assis Neves Dantas ${ }^{1}$

José Eugênio Lopes Leite ${ }^{1}$

Daniele Vieira Dantas ${ }^{1}$

Gilson de Vasconcelos Torres ${ }^{1}$

\title{
Perfil dos casos de violência socorridos por um Servico de Atendimento Móvel de Urgência Estadual
}

ABSTRACT | Introduction: External causes, particularly violence, constitute a serious challenge for public bealth. Objective: To characterize the epidemiology of violence victims assisted by a Mobile Emergency Public Care Service (SAMU). Methods: This is a descriptive, exploratory study using a quantitative approach, based on the analysis of records of service for the period between January and April,

2014. Results: Of the 211 violence incidents recorded, $41.7 \%$ involved firearms, $33.6 \%$ of cases occurred in January, $41.7 \%$ took place in the evening, $25.1 \%$ on Sundays, $85.3 \%$ had male victims, $32.2 \%$ of the victims were $15-24$ years old, $18 \%$ were under the influence of drugs, $87.2 \%$ were assisted by primary care teams, with head, face or neck accounting for $35.6 \%$ of the injuries, and public hospitals were the predominant final destination (82.5\%). Conclusion:

These findings may contribute to the planning of intersectoral actions to promote the public health, in addition to supporting strategic decisions at management level

Keywords | Violence; Prehospital care; Health profile.
RESUMO | Introdução: As causas externas, em especial as violências, constituemse um problema de saúde pública de grande magnitude e transcendência. Objetivo: Traçar um perfil epidemiológico das vítimas de violência atendidas por um Serviço de Atendimento Móvel de Urgência Estadual. Métodos: Estudo descritivo, exploratório, de abordagem quantitativa, baseado na análise das fichas de atendimento do período de janeiro a abril de 2014. Resultados: Das 211 ocorrências de violência, a maioria das vítimas $(41,7 \%)$ foram por arma de fogo; $33,6 \%$ dos atendimentos ocorreram no mês de janeiro; $41,7 \%$ aconteceram no período da noite; $25,1 \%$ no domingo; $85,3 \%$ das vítimas eram do sexo masculino; $32,2 \%$ na faixa de 15 a 24 anos; $87,2 \%$ foram atendidas por equipes de suporte básico; $35,6 \%$ das lesões aconteceram em cabeça, face ou pescoço; $18 \%$ estavam sob o uso de alguma droga; e o destino final foram os hospitais públicos (82,5\%). Conclusão: Esses resultados podem contribuir para o planejamento de ações intersetoriais de promoção à saúde coletiva, além de subsidiar decisões estratégicas úteis às esferas gestoras.

Palavras-chave | Violência; Assistência pré-hospitalar; Perfil de saúde.

\footnotetext{
${ }^{1}$ Universidade Federal do Rio Grande do Norte, Natal/RN, Brasil.
} 


\section{INTRODUÇÃO|}

Anualmente cerca de 60 milhões de pessoas sofrem algum tipo de traumatismo. Isso corresponde a uma em cada seis internações hospitalares. No Brasil, a mortalidade por trauma ocupa a terceira posição entre as causas de morte, superada apenas pelas doenças neoplásicas e cardiovasculares. Cerca de 130.000 pessoas morrem anualmente, em nosso País. No ano de 2011, no Brasil, as causas externas representaram 8,6\% do total de internações pelo Sistema Único de Saúde (SUS), com valor total pago de R\$1,02 bilhão ${ }^{1}$.

Quando se trata especificadamente da violência, esta tem se tornado alvo de todos os jornais e um fenômeno cotidiano na vida das pessoas que compartilham da mídia como fonte de informação e lazer. São homicídios, sequestros, suicídios, infanticídios, espancamentos de idosos, estupros que permeiam a sociedade atual ${ }^{2-3}$.

Segundo a Organização Mundial da Saúde (OMS), violência é definida como o uso de força física ou de poder, real ou por ameaça, contra si próprio ou outra pessoa, contra grupo ou comunidade, que resulte ou tenha qualquer possibilidade de resultar em lesão, morte, dano psicológico, deficiência de desenvolvimento ou privação ${ }^{4}$.

Um estudo abrangendo dados de 193 países-membros da OMS e dados do Banco Mundial referentes a 2012 permitiu que incapacidades físicas e Anos Potenciais de Vida Perdidos (APVP) advindos de causas violentas fossem correlacionados com as perdas econômicas correspondentes, calculadas a partir dos produtos internos brutos. O Brasil figurou em quarto lugar em anos de vida perdidos e incapacidades físicas por violência na classificação, com perdas econômicas de US\$ 9.935 bilhões nesse $\mathrm{ano}^{2}$.

Estudos sobre agravos por causas externas violentas revelam maior ocorrência em homens jovens, com uso de arma de fogo como objeto preferencial, acarretando a necessidade de tratamentos mais complexos, mais onerosos e mais demorados 5 .

Diante desse contexto, e partindo da experiência profissional dos autores, atendendo diariamente um número significativo de pacientes vítimas de violência e vivenciando o cuidado a essas vítimas no ambiente pré-hospitalar móvel de urgência, bem como no aprofundamento da leitura científica acerca deste objeto de estudo, questionou-se: qual o perfil das vítimas de violência atendidas por um serviço de atendimento móvel de urgência estadual?

Baseados nesta questão de pesquisa elaborou-se o seguinte objetivo: traçar um perfil epidemiológico das vítimas de violência atendidas por um Serviço de Atendimento Móvel de Urgência Estadual.

\section{MÉTODOS|}

Trata-se de um estudo descritivo, exploratório, de abordagem quantitativa e dados retrospectivos. O estudo descritivo pode ser compreendido como aquele em que se observa, registra, analisa, e correlaciona fatos ou acontecimentos sem manipulá-los. Descobre com precisão a frequência com que o fenômeno ocorre, sua relação e conexão com outros. Foi utilizada uma abordagem quantitativa por esta permitir a coleta sistemática de informação numérica, mediante condições de muito controle, medição e interpretação cuidadosa da realidade ${ }^{6}$.

Os dados foram coletados por meio dos registros de enfermagem utilizados nas ocorrências pelos profissionais do Serviço de Atendimento Móvel de Urgência do Estado do Rio Grande do Norte (SAMU $192 \mathrm{RN}$ ), referente ao período de $1^{\circ}$ de janeiro a 30 de abril de 2014.

Neste estudo, foram selecionadas as seguintes variáveis para categorização: dados demográficos (sexo e faixa etária), tipo de ocorrência de violência (ferimento por arma de fogo, ferimento por arma branca, agressão física e autoagressão), mês e dia do atendimento, horário da ocorrência, lesões por região corpórea, local de destino do paciente, uso de drogas e o tipo de veículo de suporte. Para estratificação da faixa etária, utilizou-se o modelopadrão da Organização Pan-Americana da Saúde (OPAS), que consiste em: menores de 1 ano; 1 a 4 anos; 5 a 14; 15 a 24; 25 a 34; 35 a 44; 45 a 54; 55 a 64; 65 a 74; e 75 e mais anos.

Como critérios de inclusão das fichas de atendimento deste estudo, definiu-se que seriam aquelas com dados preenchidos de maneira adequada e com letra legível. Como critérios de exclusão, foram as fichas com preenchimento inadequado e/ou com letra ilegível. 
A coleta de dados foi realizada nos meses de julho a setembro de 2014, a partir de um instrumento estruturado. Posteriormente, os dados foram categorizados em uma planilha do Excel e apresentados em forma de tabelas onde foram analisados por meio da estatística descritiva.

Partindo do princípio de que a pesquisa envolveu seres humanos, esta seguiu a Resolução n 466, de 12 de dezembro de 2012, do Conselho Nacional de Saúde. Além disso, salienta-se que se obteve autorização prévia da instituição para coleta dos dados, e o projeto foi aprovado pelo Comitê de Ética em Pesquisa do Hospital Universitário Onofre Lopes (HUOL) de Natal/RN, sob protocolo de $\mathrm{n}^{\circ}$ 437/2010 e CAAE: 0025.0.294.051-107.

\section{RESULTADOS}

No período de $1^{\circ}$ janeiro a 30 abril, do ano de 2014, o SAMU 192 RN atendeu um total de 3.209 ocorrências; dessas, 23 foram excluídas uma vez que estavam com seu preenchimento inadequado, insuficiente ou com letra ilegível. Portanto, considerou-se o total de 3.186 ocorrências que se encaixaram nos critérios de inclusão previamente estabelecidos. Dessas, 1.454 (45,6\%) foram traumáticas, sendo $211(14,5 \%)$ casos de violência. Segue abaixo a Tabela 1, com a distribuição dos casos de violência segundo o tipo de ocorrência.

Conforme Tabela 1 acima, dos 211 atendimentos de violência, 88 (41,7\%) foram ferimentos por arma de fogo, seguidos de 65 (30,8\%) ocorrências de agressão física, 49 $(23,2 \%)$ de ferimentos por arma branca e 09 (4,3\%) de autoagressão. Com relação ao mês da ocorrência e o horário do atendimento, com $71(33,6 \%)$ atendimentos prevaleceu o mês de janeiro, seguido do mês de abril $(27,0 \%)$, no horário da noite 88 (41,7\%).

Os dados desta pesquisa também revelaram que o dia da semana que mais apresentou ocorrências de casos de violência foi o domingo 53 (25,1\%); seguidos de 43 (20,4\%), no sábado; e 31 (14,7\%), na sexta-feira.

Nos atendimentos dos casos de violência realizados pelo SAMU 192 RN, 180 (85,3\%) eram do sexo masculino, enquanto $31(14,7 \%)$ foram do sexo feminino.
Tabela 1 - Ocorrências de violência atendidas pelo SAMU 192 $\mathrm{RN}$ segundo o tipo de ocorrência, mês e horário do atendimento. Rio Grande do Norte, 2014

\begin{tabular}{lcc}
\hline Variáveis & N & $\%$ \\
\hline Tipo de ocorrência & 88 & 41,7 \\
\hline FAF* $^{* *}$ & 49 & 23,2 \\
FAB $^{* *}$ & 65 & 30,8 \\
Agressão física & 9 & 4,3 \\
Autoagressão & & \\
\hline Mês da ocorrência & 71 & 33,6 \\
Janeiro & 42 & 19,9 \\
Fevereiro & 41 & 19,4 \\
Março & 57 & 27,0 \\
Abril & & \\
\hline Horário do atendimento & 45 & 21,3 \\
Das 0h às 05h59 & 30 & 14,2 \\
Das 6h às 12h & 48 & 22,7 \\
Das 12h01às 18h & 88 & 41,7 \\
Das 18h01 às 23h59 & $\mathbf{2 1 1}$ & $\mathbf{1 0 0 , 0}$ \\
\hline Total & & \\
\hline
\end{tabular}

*Ferimento por arma de fogo; ** Ferimento por arma branca.

Quando analisamos o sexo por tipo de ocorrência, a predominância do sexo masculino prevalece em todos os tipos (Tabela 2). Ao observar a faixa etária, 68 (32,2\%) ocorreram na faixa de 15 a 24 anos, seguido de 63 (29,9\%) na faixa de 25 a 34 anos, e $36(17,1 \%)$ entre 35 e 44 anos de idade. Ao analisar por tipo de ocorrência, observa-se semelhante predomínio nessas faixas etárias.

Conforme Tabela 3, $184(87,2 \%)$ das ocorrências foram atendidas por equipes de suporte básico, formadas por técnico de enfermagem e condutor socorrista.

Quando se observa os locais onde ocorreram as lesões, detecta-se que $96(35,6 \%)$ das lesões aconteceram em cabeça, face ou pescoço, seguido de 88 (32,6\%) de lesões em membros ou cintura pélvica.

Sobre o consumo de drogas, 38 (18\%) pacientes apresentavam sinais de uso de alguma droga lícita ou ilícita, sendo em sua maioria o álcool. 
Tabela 2 - Ocorrências de violência atendidas pelo SAMU $192 \mathrm{RN}$ segundo o sexo e faixa etária por tipo de ocorrência. Rio Grande do Norte, 2014

\begin{tabular}{|c|c|c|c|c|c|c|c|c|c|c|}
\hline \multirow{2}{*}{$\begin{array}{l}\text { Variáveis } \\
\text { Sexo }\end{array}$} & \multicolumn{2}{|c|}{ FAF } & \multicolumn{2}{|c|}{ FAB } & \multicolumn{3}{|c|}{ Agressão física } & \multicolumn{2}{|c|}{ Autoagressão } & \multirow{2}{*}{$\begin{array}{r}\text { Total } \\
\%\end{array}$} \\
\hline & $\mathbf{N}$ & $\%$ & $\mathbf{N}$ & $\%$ & $\mathbf{N}$ & $\%$ & $\mathbf{N}$ & $\%$ & $\mathbf{N}$ & \\
\hline Masculino & 79 & 89,8 & 42 & 85,7 & 53 & 81,5 & 6 & 66,7 & 180 & 85,3 \\
\hline Feminino & 9 & 10,2 & 7 & 14,3 & 12 & 18,5 & 3 & 33,3 & 31 & 14,7 \\
\hline Total & 88 & 100,0 & 49 & 100,0 & 65 & 100,0 & 9 & 100,0 & 211 & 100,0 \\
\hline \multicolumn{11}{|c|}{ Faixa etária } \\
\hline$\leq 14$ & 0 & 0,0 & 1 & 2,0 & 1 & 1,5 & 0 & 0,0 & 2 & 0,9 \\
\hline $15|-| 24$ & 41 & 46,6 & 11 & 22,4 & 13 & 20,0 & 3 & 33,3 & 68 & 32,2 \\
\hline $25|-| 34$ & 21 & 23,9 & 19 & 38,8 & 23 & 35,4 & 0 & 0,0 & 63 & 29,9 \\
\hline $35|-| 44$ & 12 & 13,6 & 8 & 16,3 & 13 & 20,0 & 3 & 33,3 & 36 & 17,1 \\
\hline $45|-| 54$ & 6 & 6,8 & 4 & 8,2 & 7 & 10,8 & 2 & 22,2 & 19 & 9,0 \\
\hline $55|-| 64$ & 1 & 1,1 & 2 & 4,1 & 4 & 6,2 & 0 & 0,0 & 7 & 3,3 \\
\hline$\geq 65$ & 0 & 0,0 & 1 & 2,0 & 1 & 1,5 & 0 & 0,0 & 2 & 0,9 \\
\hline $\mathrm{N} / \mathrm{l}$ & 7 & 8,0 & 3 & 6,1 & 3 & 4,6 & 1 & 11,1 & 14 & 6,6 \\
\hline Total & 88 & 100,0 & 49 & 100,0 & 65 & 100,0 & 9 & 100,0 & 211 & 100,0 \\
\hline
\end{tabular}

${ }^{*}$ Ferimento por arma de fogo; **Ferimento por arma branca.

Tabela 3 - Ocorrências de violência atendidas pelo SAMU $192 \mathrm{RN}$ segundo o tipo de transporte, lesões por região corpórea e uso de drogas. Rio Grande do Norte, 2014

\begin{tabular}{lcc}
\hline Variáveis & N & $\%$ \\
\hline Tipo de viatura & 184 & 87,2 \\
Unidade de Suporte Básico & 27 & 12,8 \\
\hline Unidade de Suporte Avançado & & \\
\hline Lesões por região corpórea & 96 & 35,6 \\
\hline Cabeça/Face/pescoço & 57 & 21,1 \\
Tórax & 29 & 10,7 \\
Abdome & 88 & 32,6 \\
\hline Membros/Cintura pélvica & & \\
\hline Uso de drogas & 38 & 18,0 \\
\hline Sim & 173 & 82,0 \\
\hline Não & $\mathbf{2 1 1}$ & $\mathbf{1 0 0 , 0}$ \\
\hline Total & &
\end{tabular}

Conforme Tabela 4, o destino final da maioria das ocorrências de casos de violência foram os hospitais públicos, com 174 (82,5\%) pacientes, seguido dos óbitos em $24(11,4 \%)$ casos.

Tabela 4 - Ocorrências de violência atendidas pelo SAMU 192 RN segundo o destino do paciente. Rio Grande do Norte, 2014

\begin{tabular}{lcc}
\hline Variável & N & $\%$ \\
\hline Destino do paciente & 174 & 82,5 \\
Hospital Públicos & 1 & 0,5 \\
Hospital Privados & 4 & 1,9 \\
UPAs & & \\
Óbitos & 24 & 11,4 \\
Outros* & 8 & 3,8 \\
\hline Total & $\mathbf{2 1 1}$ & $\mathbf{1 0 0 , 0 \%}$ \\
\hline
\end{tabular}

\#Unidades de Pronto Atendimento; *Apoio de mais de uma viatura no local ou orientação sem remoção. 


\section{DISCUSSÃO|}

As ocorrências realizadas com vítimas de violência permitem aos pesquisadores investigar uma série de variáveis importantes para o diagnóstico de um perfil epidemiológico.

Com relação ao tipo de ocorrência prevalecer o uso de armas de fogo, pode-se inferir que esse cenário pode ser explicado pelo fato de que, em 2005, o Senado Federal encaminhou à Câmara dos Deputados um projeto de lei que proibiria o comércio de armas no Brasil e marcou um plebiscito para outubro do mesmo ano, com o intuito de diminuir as crescentes taxas de homicídios verificadas no País. Sendo assim, no Brasil, onde apenas 10\% das armas tinham registro, o Estatuto do Desarmamento previa que o porte ilegal fosse tratado como crime inafiançável e a pena por esta infração poderia chegar a 12 anos. Entretanto, o plebiscito não permitiu que a lei entrasse em vigor, pois defensores do porte de armas argumentaram que elas eram a defesa do cidadão, e a privação delas era o mesmo que deixá-lo à mercê dos bandidos 5 .

Dados semelhantes foram constatados por estudiosos ${ }^{8}$, pesquisando a violência em serviço de emergência do Rio Grande do Sul (RS). Com relação aos meios de agressão, das agressões especificadas, 100 pacientes foram vitimados por objeto penetrante $(26 \%)$, seguidos de 27 por objeto cortante $(7 \%)$ e 19 por objeto contundente $(4,9 \%)$. O uso da força corporal/espancamento representou 59 pacientes $(15,4 \%)$, tendo menor escala as agressões por coronhada, com nove casos $(2,2 \%)$, e duas situações de queimadura $(0,5 \%)$. Nos casos em que foi utilizada arma, a pesquisa identificou: 93 feridos por arma de fogo $(77,5 \%)$ e 27 por arma branca $(22,5 \%)$.

Diferentemente, pesquisadores ${ }^{9}$ encontraram que o tipo de violência mais frequente foi a física $(93,6 \%)$ com o meio de agressão mais utilizado sendo a força corporal/ espancamento $(80,7 \%)$.

No que tange ao mês da ocorrência ter sido janeiro, especialistas $^{10}$ da área do Atendimento Pré-Hospitalar (APH) no Brasil, em uma pesquisa realizada por um Serviço de Atendimento Móvel de Urgência (SAMU) metropolitano, indicaram o mês de janeiro foi o que mais apresentou ocorrências para o serviço em estudo, com 771 $(18,84 \%)$ dos atendimentos.
Possivelmente, o predomínio de atendimentos de emergência ocorre em janeiro por ser esse um mês de férias, na estação do verão brasileiro, onde acontecem muitas festas e uso de bebidas alcoólicas que favorecem a ocorrência de atos violentos.

Já estudiosos ${ }^{9}$, em pesquisa sobre violência interpessoal, encontraram que o período do ano em que predominaram ocorrências de violência foi o mês de fevereiro $(12,8 \%)$ e no horário compreendido entre as $18 \mathrm{~h} 01 \mathrm{e}$ as $24 \mathrm{~h}(57,8 \%)$.

Os mesmos investigadores, em estudo realizado em sete municípios do estado de São Paulo/SP, também identificaram o domingo como o dia da semana mais violento $(16,5 \%)^{9}$.

As ocorrências de violência no turno da noite também prevaleceram em outros estudos ${ }^{8}$, quando pesquisavam este fenômeno em um serviço de emergência do sul brasileiro, e perceberam que o turno da noite foi responsável por 223 $(58,1 \%)$ registros de ocorrência de violência. Os turnos da manhã e tarde apresentaram percentuais semelhantes, de $81(21,1 \%)$ e $80(20,8 \%)$, respectivamente.

Com relação ao sexo e a faixa etária das vítimas de violência, analisando 453 prontuários de adolescentes internados em decorrência de causas externas em um serviço de emergência em Recife/PE, outros estudiosos ${ }^{11}$ encontraram 64 casos de agressão por arma de fogo a vítimas do sexo masculino e apenas 6 do feminino. As armas brancas foram responsáveis por 21 hospitalizações em adolescentes masculinos e 6 em femininas.

Estudo $^{12}$ realizado sobre perfil de vítimas de agressão com armas em três Hospitais de Campo Grande/MS detectou que, dos 78 prontuários analisados, 71 (91\%) eram de adolescentes do sexo masculino e $7(8,9 \%)$ do feminino. Dos adolescentes masculinos, 46 (58,9\%) foram vítimas de ferimentos por armas de fogo e $25(32,1 \%)$ sofreram lesões por armas brancas. Entre as adolescentes, $6(85,7 \%)$ sofreram lesões por armas brancas e 1 (14,3\%) foi agredida com arma de fogo.

Estudo $^{8}$ em um serviço de emergência do Hospital Cristo Redentor (HCR), em março e abril de 2010, apresentou que 384 atendimentos de emergência foram prestados às vítimas de violência física em pessoas com idade igual ou superior a 18 anos. Quanto à faixa etária das vítimas, a pesquisa identificou a oscilação entre 18 e 68 anos 
(observando critério de inclusão), com maior incidência entre 18 e 29 anos, seguido de 30 a 39 anos, para ambos os sexos. Na amostra estudada, evidenciou-se que $275(71,6 \%)$ dos pacientes atendidos eram do sexo masculino.

Em investigação quanto aos atendimentos de emergência por violência em serviços públicos de Teresina/PI, a faixa etária mais afetada foi a de 20 a 29 anos (40,9\%), seguida pela de 30 a 39 anos $(17,5 \%)^{13}$.

Constata-se maior vulnerabilidade, morbidade e mortalidade em jovens do sexo masculino, por causas externas, em especial pela violência, com uso da arma de fogo.

No que se refere ao tipo de viatura mais utilizada, achados semelhantes foram identificados por um estudo realizado em Catanduva/SP, onde a Unidade de Suporte Básico (USB) foi a responsável por $90 \%$ dos atendimentos ${ }^{14}$.

Corroborando com os resultados desta pesquisa, outros estudiosos ${ }^{10}$ também afirmaram que, no que se refere ao tipo de veículo de suporte utilizado para o atendimento das 4.092 ocorrências realizadas no período estudado, destaca-se a apresentação das USBs, que atendem pacientes de menor gravidade, em 3.930 (96,04\%) dos casos de atendimentos. Já as Unidades de Suporte Avançado (USA), utilizadas para a assistência de paciente mais graves, foram usadas em apenas 162 (3,96\%) atendimentos.

Pesquisadores $^{8}$, analisando o perfil de violência em um Hospital no Rio Grande do Sul, detectaram resultados semelhantes aos nossos no que se refere a lesões por região corpórea, em que, ao analisar as partes do corpo lesadas, as mais atingidas foram: cabeça e pescoço, com 241 casos (62,8\%); tronco (abdome, tórax, pelve e dorso), com 120 (31,3\%); e membros superiores, com 119 (31\%); os membros inferiores representaram 63 ocorrências $(16,4 \%)$.

Alguns autores ${ }^{9}$ também encontraram que as partes do corpo mais atingidas pela agressão foram a cabeça/face $(47,7 \%)$ e os membros superiores (20,18\%), sendo a primeira mais comum nas mulheres, e a segunda, nos homens.

O projeto Viva mostrou que cabeça e face $(49,6 \%)$, membros superiores $(31,4 \%)$, tórax e dorso $(18,2 \%)$ foram as partes mais acometidas em relação à localização da lesão ${ }^{15}$.

Diferentemente, outro estudo ${ }^{12}$, analisando as lesões por arma de fogo em adolescentes de Campo Grande/MS, detectou que as lesões em tórax (43,5\%), lesões múltiplas $(39,7 \%)$ e abdominais $(26,9 \%)$ foram as mais frequentes.

Pesquisa realizada em Pouso Alegre mostra que, nas agressões, a cabeça e os membros superiores passam a ter grande importância $(37,3 \%$ e $34,3 \%$, respectivamente), por serem regiões bastante expostas a situações de violência - a cabeça, por ser visada pelo agressor, e os membros superiores, pela utilização de autodefesa ${ }^{16}$.

Sobre a presença de sinais do uso de drogas nos pacientes atendidos, um estudo realizado na região do primeiro Consórcio Intermunicipal de Saúde do Brasil (CISA), no interior de São Paulo, suspeitou-se do uso de álcool pelo agressor em $35,8 \%$ dos casos, mas a maior parte das vítimas $(55,0 \%)$ informou não fazer uso da substância 9 .

Estudo realizado em três Unidades Básicas de Saúde da Região Nordeste brasileiro, apontou a percepção de 30 profissionais de saúde sobre os fatores que influenciam as situações de violência. Os profissionais expressaram como principais causas o machismo, as condições econômicas, o alcoolismo e os antecedentes familiares de violência. Assim, estudiosos argumentam que o uso de álcool e de outras drogas pode estar presente em casos de violência, tornando o enfrentamento desta, bem como a prevenção e a assistência às vítimas que vivenciam esta situação, ainda mais complexo e multidimensional ${ }^{17}$.

Desse modo, o abuso de álcool e outras drogas frequentemente têm um papel importante nas atitudes violentas. No entanto, trata-se de uma articulação complexa que envolve diversos aspectos, indo de encontro à designação causal. Seu papel específico não está claro, ou seja, é difícil determinar com precisão o nexo causal entre o uso de substâncias psicoativas e atos violentos; as influências do meio e as características individuais de usuários de álcool e drogas; a prevalência e as correlações precisas entre elas. $\mathrm{O}$ fenômeno da violência e a articulação com as drogas exige que estes temas sejam tratados com instrumentos, conhecimentos e ações que ultrapassem o moralismo simplista. Dessa forma, há necessidade de se distinguir a complexidade do contexto, a dinâmica social, normas culturais historicamente construídas - a questão de gênero, de fatores de personalidade e individualidade ${ }^{18}$.

Analisando o destino dos pacientes, após estabilização hemodinâmica dos mesmos, os achados desta pesquisa não condizem com a estruturação da Rede de Atenção às 
Urgências (RAU). De acordo com o Ministério da Saúde, as Unidades de Pronto Atendimento (UPAs) devem funcionar como unidades intermediárias entre as Unidades Básicas de Saúde (UBSs) e os hospitais, atuando de forma integrada com o SAMU. Considerando que $83 \%$ das ocorrências destinaram-se aos hospitais, é possível compreender que a estruturação da rede está deficiente, impossibilitando a continuidade da atenção, ratificando uma tendência hospitalocêntrica ${ }^{19}$.

Para estudioso da área de saúde coletiva ${ }^{20}$, esses resultados devem-se à fragmentação dos sistemas de atenção à saúde, que se volta às condições agudas e às agudizações de condições crônicas, o que não é coerente com as situações atuais de saúde onde há o declínio dos problemas agudos e o aumento das condições crônicas.

\section{CONCLUSÃO|}

Considerando os objetivos propostos para este estudo, os resultados encontrados permitiram concluir que, no período estudado, o SAMU $192 \mathrm{RN}$ atendeu um total de 3.186 ocorrências. Dessas, 1.454 (45,6\%) foram traumáticas, sendo $211(14,5 \%)$ casos de violência.

Em relação ao perfil epidemiológico dos casos de violência, tem-se que $88(41,7 \%)$ foram ferimentos por arma de fogo; $71(33,6 \%)$ atendimentos ocorreram no mês de Janeiro; 88 $(41,7 \%)$ dos atendimentos de violência aconteceram no período da noite; $53(25,1 \%)$ dos atendimentos ocorreram no domingo; 180 (85,3\%) eram do sexo masculino; 68 $(32,2 \%)$ ocorreram na faixa de 15 a 24 anos; $184(87,2 \%)$ das ocorrências foram atendidas por equipes de suporte básico (técnico de enfermagem e condutor); 96 (35,6\%) lesões aconteceram em cabeça, face ou pescoço; 38 (18\%) estavam sob o uso de alguma droga; destino final da maioria das ocorrências de violência foram os hospitais públicos com $174(82,5 \%)$ pacientes.

Com relação às ocorrências demandadas pelo serviço em análise, chama a atenção as inúmeras fichas de atendimento com dados preenchidos de maneira inadequada ou com letra ilegível durante a realização deste estudo, o que reflete uma limitação dele.

Conclui-se que esses resultados podem contribuir para o planejamento de ações intersetoriais de promoção à saúde, incluindo dimensionamento de pessoal e alocação e otimização de recursos, além de subsidiar decisões estratégicas úteis à esfera gestora estadual. Além disso, estudos desta natureza permitem sugerir ênfase em treinamentos das equipes do $\mathrm{APH}$ que priorizem aspectos referentes à cinemática do trauma, incluindo a remoção de vítimas em quadros de difícil acesso e procedimentos orientados pelos protocolos do trauma. Esperamos que os resultados alcançados impulsionem a realização de novos estudos.

\section{REFERÊNCIAS |}

1. Brasil. Ministério da Saúde. Manual instrutivo da Rede de Atenção às Urgências e Emergências no Sistema Único de Saúde (SUS). Brasília: Ministério da Saúde; 2013.

2. Dantas RAN, Farias GM, Rocha KMM, Costa IKF, Medeiros RC. The impact of violence in public health: literature review. Rev Enferm UFPE On Line [Internet]. 2010 [acesso em 12 ago 2014]; 4(esp):1935-43. Disponível em: URL: <http://www.revista.ufpe.br/revistaenfermagem/ index.php/revista/article/view/1480/pdf_253>.

3. Albuquerque NMG, Cavalcante CAA, Macêdo MLAF, Oliveira JSA, Medeiros SM. Causas externas: características de crianças e adolescentes assistidas em um hospital do Rio Grande do Norte. Rev Bras Pesq Saúde [Internet]. 2014 [acesso em 12 ago 2014]; 16(2):7-14. Disponível em: URL: <http://periodicos.ufes.br/RBPS/article/ viewFile/9297/6471>.

4. Mascarenhas MDM, Malta DC, Silva MMA, Lima CM, Carvalho MGM, Oliveira VLA. Violência contra a criança: revelando o perfil dos atendimentos em serviços de emergência, Brasil, 2006 e 2007. Cad Saúde Pública [Internet]. 2010 [acesso em 07 ago 2015]; 26(2):347-57. Disponível em: URL: <http://www. scielo.br/scielo.php? script=sci_arttext\&pid=S0102311X2010000200013\&lng=en $>$.

5. Costa IKF. Riscos ocupacionais e acidentes de trabalho em um Serviço de Atendimento Móvel de Urgência do Rio Grande do Norte. Natal. Dissertação [Mestrado em Enfermagem] - Universidade Federal do Rio Grande do Norte; 2011. 
6. Cervo AL, Bervian PA, Silva R. Metodologia científica. 6. ed. São Paulo: Pearson Prentice Hall; 2007.

7. Conselho Nacional de Saúde (Brasil). Resolução nº. 466, de 12 de dezembro de 2012. Diretrizes e Normas Regulamentadoras de Pesquisa Envolvendo Seres Humanos [Internet]. Diário Oficial da União 13 jun 2013 [acesso em 30 ago 2014]. Disponível em: URL: <http:// conselho.saude.gov.br/resolucoes/2012/Reso466.pdf>

8. Dalbem GG, Unicovsky MAR. A natureza das lesões traumáticas ocasionadas pela violência física em pacientes atendidos em serviço de emergência de um hospital público. Enfermagem em Foco [Internet]. 2012 [acesso em 07 ago 2015]; 2(3):81-8. Disponível em: URL: <http:/ / revista.portalcofen.gov.br/index.php/enfermagem/ article/view/260/148>.

9. Cecilio LPP, Garbin CAS, Rovida TAS, Queiróz APDG, Garbin AJI. Violência interpessoal: estudo descritivo dos casos não fatais atendidos em uma unidade de urgência e emergência referência de sete municípios do estado de São Paulo, Brasil, 2008 a 2010. Epidemiol Serv Saúde [Internet]. 2012 [acesso em 07 ago 2015]; 21(2):293-304. Disponível em: URL: <http://scielo.iec.pa.gov.br/scielo.php?script=sci_ arttext\&pid=S1679-49742012000200012\&lng=pt $>$.

10. Dantas RAN, Costa IKF, Nóbrega WG, Dantas DV, Costa IKFC, Torres GV. Ocorrências realizadas pelo serviço de Atendimento Móvel de Urgência Metropolitano. Rev Enferm UFPE On Line [Internet]. 2014 [acesso em 07 ago 2015]; 8(4):842-9. Disponível em: URL: <http://www. revista.ufpe.br/revistaenfermagem/index.php/revista/ article/download/4077/8811>.

11. Barbosa HSC, Bezerra SMMS, Lyra DM, Acioli EE, Oliveira LS. Perfil e fatores associados a morbi-mortalidade por causas externas de adolescentes atendidos em um serviço de emergência em Recife entre 2004 e 2005. Rev Enferm UFPE On Line [Internet]. 2007 [acesso em 07 ago 2015]; 1(2):173-80. Disponível em: URL: <http://www. revista.ufpe.br/revistaenfermagem/index.php/revista/ article/download/379-8806-1-/1232>.

12. Silva APS, Pontes ERJC, Tognini JRF. Epidemiological profile and hospitalization costs of cases of assault with weapons among adolescents in Campo Grande, MS, Brazil. Rev Pesq: Cuid Fundam Online [Internet]. 2012 [acesso em 07 ago 2015]; 4(3):2493-01. Disponível em: URL: <http:/ / www.seer.unirio.br/index.php/cuidadofundamental/ article/view/1856>.

13. Mascarenhas MDM, Pedrosa AAG. Atendimentos de emergência por violência em serviços públicos de Teresina, PI. Rev Bras Enferm [Internet]. 2008 [acesso em 07 ago 2015]; 61(4):493-9. Disponível em: URL: <http://www. scielo.br/scielo.php? script $=$ sci_arttext\&pid $=$ S0034$71672008000400016 \& \operatorname{lng}=\mathrm{en}>$.

14. Gonsaga RAT, Brugugnolli ID, Zanutto TA, Gilioli JP, Silva LFC, Fraga GP. Características dos atendimentos realizados pelo Serviço de Atendimento Móvel de Urgência no município de Catanduva, Estado de São Paulo, Brasil, 2006 a 2012. Epidemiol Serv Saúde [Internet]. 2013 [acesso em 07 ago 2015]; 22(2):317-24. Disponível em: URL: <http:/ / scielo. iec.pa.gov.br/scielo.php?script=sci_arttext\&pid=S167949742013000200013\&lng $=$ pt\&nrm $=$ iso\&tlng $=$ pt $>$.

15. Andrade SSCA, Sá NNB, Carvalho MGO, Lima CM, Silva MMA, Moraes Neto OL, et al. Perfil das vítimas de violências e acidentes atendidas em serviços de urgência e emergência selecionados em capitais brasileiras: vigilância de violências e acidentes, 2009. Epidemiol Serv Saúde [Internet]. 2012 [acesso em 07 ago 2015]; 21(1):21-30. Disponível em: URL: <http://scielo.iec. pa.gov.br/scielo.php? script $=$ sci_arttext\&pid $=$ S167949742012000100003\&lng=pt>.

16. Mesquita Filho M, Jorge MHPM. Características da morbidade por causas externas em serviço de urgência. Rev Bras Epidemiol [Internet]. 2007 [acesso em 07 ago 2015]; 10(4):579-91. Disponível em: URL: <http://www. scielo.br/scielo.php? script $=$ sci_arttext\&pid $=$ S1415790X2007000400016\&lng=en>

17. Marinheiro ALV, Vieira EM, Souza L. Prevalência da violência contra a mulher usuária de serviço de saúde. Rev. Saúde Pública [Internet]. 2006 [acesso em 07 ago 2015]; 40(4):604-10. Disponível em: URL: <http://www. scielo.br/scielo.php? script=sci_arttext\&pid=S0034$89102006000500008 \& \operatorname{lng}=\mathrm{en}>$.

18. Vieira, LB, Cortes LF, Padoin SMM, Souza IEO, Souza CCP, Terra MG. Abuso de álcool e drogas e violência contra as mulheres: denúncias de vividos. Rev Bras Enferm. [Internet]. 2014 mai-jun [acesso em 07 ago 2015];67(3):36672. Disponível em: URL: http://www.scielo.br/pdf/ reben/v67n3/0034-7167-reben-67-03-0366.pdf 
19. Brasil. Ministério da Saúde. Secretaria de Vigilância em Saúde. Departamento de Análise de Situação em Saúde. Saúde Brasil 2011: uma análise da situação de saúde e a vigilância da saúde da mulher. Brasília: Ministério da Saúde; 2012.

20. Mendes EV. As redes de atenção à saúde. Minas Gerais: Secretaria de Estado de Saúde de Minas Gerais; 2010.

Correspondência para/Reprint request to:

Rodrigo Assis Neves Dantas

Rua Petra Kelly, 61, Geraldo Galvão Residencial, casa 48,

Nova Parnamirim, Parnamirim/RN, Brasil

CEP: 59152-330

E-mail: rodrigoenf@yahoo.com.br

Submetido em: 07/08/2015

Aceito em: 21/12/2015 